\title{
Adverse childhood experiences and the association with initiating Alcohol, and chat Consumption before age of 18, in school going adolescents, Ethiopia: cross sectional study
}

Mekonnen Tsehay ( $\sim$ jimma1760@gmail.com )

Wollo University https://orcid.org/0000-0002-7652-5212

Mogese Necho

Wollo University

Asmare Belete

Wollo University

\section{Research}

Keywords: alcohol, chat, adverse childhood experience, social support, adolescent

Posted Date: April 28th, 2020

DOI: https://doi.org/10.21203/rs.3.rs-24816/v1

License: (c) (i) This work is licensed under a Creative Commons Attribution 4.0 International License.

Read Full License 
Adverse childhood experiences and the association with initiating Alcohol, and chat Consumption before age of 18, in school going adolescents, Ethiopia: cross sectional study

\section{Mekonnen Tsehay ${ }^{1}$, Mogesie Necho $^{2}$, ASmare Belete $^{3}$}

${ }^{1}$ Mekonnen Tsehay, Department of psychiatry, college of medicine and health science, Wollo University, Dessie, Ethiopia

Email: jimma1760@gmail.com

${ }^{2}$ Mogese Necho, department of psychiatry, college of medicine and health science, Wollo University, Dessie, Ethiopia

Email: nechomoges2014@gmail.com

${ }^{3}$ Asmare Belete. Department of psychiatry, college of medicine and health science, Wollo University, Dessie, Ethiopia

Email: yasmarebel@yahoo.com

*Corresponding author:

Mekonnen Tsehay, Department of psychiatry, college of medicine and health science, Wollo University, Dessie, Ethiopia

Email: jimma1760@gmail.com, pobox: 1145 


\begin{abstract}
Background: Adverse childhood experiences (ACEs) were associated with poor health outcomes and health-threatening behaviors later in life. The objective of the present study was to investigate associations between adverse childhood experiences (ACEs) and early initiation of alcohol and chat use among school going adolescents.
\end{abstract}

Methods: A cross-sectional study was performed with 546 school going adolescents. The ACE International Questionnaire (ACE-IQ) was used to assess ACEs, including emotional, physical, and sexual forms of abuse and neglect, as well as household dysfunction. Alcohol and chat use was assessed by questions prepared by authors. Multiple logistic regression models were used to examine the associations between overall ACE score and alcohol use and the potential moderating effects of confounders.

Results: prevalence of chat chewing behavior was $26.6 \%$ followed by alcohol use $20 \%$, and small number of student's use cigarette smoking and other illicit drug users $(0.9 \%$ ) (ganja and Shisha) each. A total of $66.2 \%$ of participants reported at least one ACE, and $5.93 \%$ reported four or more ACEs. Increased ACE scores were associated with increased risks of drinking (adjusted odds ratio $[\mathrm{AOR}]=1.09,95 \%$ confidence intervals $[\mathrm{CI}]: 1.00-1.09$ ), chronic disease $(\mathrm{AOR}=1.17,95 \% \mathrm{CI}: 1.06-1.28)$, depression $(\mathrm{AOR}=1.37,95 \% \mathrm{CI}: 1.27-1.48)$, and posttraumatic stress disorder $(\mathrm{AOR}=1.32,95 \% \mathrm{CI}: 1.23-1.42)$ in adulthood. After adjusting for confounding factors, the individual ACE components had different impacts on risk behavior and health, particularly on poor mental health outcomes in adulthood.

Conclusions: Alcohol and Chat use was prevalent and public health concern among school going adolescents. ACEs were significantly associated with risk behaviors, alcohol and chat use may lead poor health and educational outcomes among adolescent students and numbers of ACEs have graded association. Social support, sex, residence, parent educational status and current level of depressive symptoms were significant modulating factors, which parents, school teachers, psychologists and adolescent health care providers should give concern to decrease the effect on school going adolescents.

Key word: alcohol, chat, adverse childhood experience, social support, adolescent 


\section{INTRODUCTION}

Alcohol has a complex role in Ethiopia society. While many of Ethiopian adults drink alcohol at levels that increase their risk of alcohol-related harm[1]. In many countries, including Ethiopia, alcohol is responsible for a considerable burden of death, disease, and injury; and alcohol-related harm also affects families, specifically children[2].

Research shows that, burden attributable to substance use increases substantially in adolescence and young adulthood[3]. Alcohol have short-term and long term consequences for adolescents such as injury, violence, self-harm, and risky sexual behavior and Alcohol use during early adolescence is a major risk factor for later alcohol abuse and dependence [4, 5].

The use of khat (Catha edulis), cigarettes, heroine, alcohol and other substances is a worldwide threat which especially affects young people[6]. The World Health Organization (WHO) estimates that there are about 2 billion people worldwide who consume alcoholic beverages, of whom 76.3 million are affected by alcohol-related disorders[7].

Excessive drinking may also have lasting effects on adolescent brain development $[8,9]$. Therefore, this research that improves our understanding of the risk factors for adolescent drinking is needed to inform policy aimed at improving the health and social outcomes for young Ethiopian.

The overall prevalence of 'any substance use' among adolescents in sub-Saharan Africa was 41.6\%, with the highest rate in Central Africa at 55.5\%[10]. This meta-analysis study also reported that, the use of caffeine-containing products (including coffee or kola nut) was most predominant at $41.2 \%$, followed by alcohol at $32.8 \%$, tobacco products $23.5 \%$, khat $22.0 \%$ and cannabis $15.9 \%$. Other abused substances included depressants at $11.3 \%$, amphetamines $9.4 \%$, heroin $4.0 \%$ and cocaine $3.9 \%$.

Adverse childhood experiences (ACEs) are traumatic events occurring before age 18. Adverse childhood experiences (sometimes referred to as ACEs) are potentially traumatic events that can have negative, long lasting effects on health and well-being. These experiences range from physical, emotional, or sexual abuse to parental divorce or the incarceration of a parent or guardian [11-14].

The prevalence of any adverse childhood event ranged from $7.9 \%$ to $32 \%$ among women and $20 \%$ to $24 \%$ among men[14, 15].and over all prevalence was estimated as $36.3 \%$ in worldwide[16].

World Health Organization multicultural study in women reported, In Ethiopia, Prevalence of partner violence Nearly one half (49\%) of ever-partnered women experienced physical violence by a partner at some point in their lives, and $29 \%$ during the past 12 months. $59 \%$ of everpartnered women experienced sexual violence at some point, and $44 \%$ during the past 12 months. Combining the data for physical and sexual violence, $71 \%$ of ever-partnered women 
experienced one or the other form of violence, or both, over their lifetime. 35\% of all everpartnered women experienced at least one severe form of physical violence[17].

Childhood development has effects on health and well-being throughout life[18]. ACEs lead to poor health outcomes and health-threatening behaviors. High ACE scores in the first eighteen years of life are linked to poor outcomes in physical and mental health and social functioning.

The ACE study described adverse experiences as "common, stressful and traumatic exposures affecting the (neuro) development of children"[19]. The hypothesis posits that stressful childhood experiences such as abuse and neglect, or growing up in a dysfunctional household, create common vulnerability to social, emotional and cognitive impairments that lead to increased risk of poor health behavior, social adjustment and physical and mental illness.

One mechanism by which adverse childhood experiences result in long-term health consequences is by engaging in adult health-risk-taking behaviors[20]. These include alcohol and drug abuse, having multiple sex partners, cigarette smoking, and compulsive eating leading to Obesity[21-24]. Individual's attempt such behaviors as coping and self-regulating the experiences of emotional pain, anxiety, anger and/or depression related to unresolved adverse childhood experiences[25].

Identifying factors that alter the processing of stressful events following exposure to ACEs may be a valuable tool in developing interventions aimed at preventing or mitigating the long-term health consequences of ACEs[26].

Social support may have a protective or buffering effect against the consequences of a stressful event by enhancing cognitive and emotional processing of the experience and protect people from going to substance uses behaviors[27].

Although ACEs are more prevalent in low- and middle-income countries, including Ethiopia owing to limited resources and less social health care [28], little is known about their health and risky behaviors impacts. For example, there is limited information on the differential effects of individual ACE components on alcohol and chat use in Ethiopia.

The primary objective of the current study was to examine the relationships between individual three ACE types (abuse, neglect and household dysfunction) and high-risk health behaviors in adolescents in Ethiopia. In addition, we examined factors that modify the association between three ACE types (abuse, neglect and household dysfunction) and high-risk health behaviors in adolescents in Ethiopia. 


\section{Methods and materials}

\section{Study Design and study Setting}

The school-based cross-sectional study design was employed from April 2/2018 -May 30/ 2018 G.C. This study was conducted in Jimma town government and private secondary school students. Jimma is located at $357 \mathrm{~km}$ to the southwest of Addis Ababa. It is found in the Oromia Region of Ethiopia. Projecting from 2007 census Jimma town has a total population of 159,009 of whom 80,897 where males and 78,112 were females.

The town has six public and eight private high school's 9383 students had registered for grades 9, 10, 11 and 12 in the academic year of 2017/18. Of these, 7292 were from public and 2091 were from private schools.

\section{Study population}

All adolescent students aged 10-19 who were studying in selected Jimma town secondary schools and students who are willing and allowed by the parents to participate in the study were recruited. Adolescents found to have severe illness during the study and difficulties in communication to the data collectors were excluded.

\section{Sampling procedures}

The sample was calculated by taking the prevalence of alcohol use among high school students of eastern Ethiopia which were $22.2 \%$, for the sake of to get large sample size[29]. Assuming any particular outcome to be within a 5\% margin of error and a 95\% confidence interval of certainty (alpha $=0.05$ ), design effect $=2$ and a $10 \%$ nonresponse rate. Based on these assumptions the sample size of the study was calculated using a single proportion formula and the final sample size was 583.

A multistage sampling technique was used. In the first stage, schools were stratified into two. all the government schools $(n=6)$ and private schools $(n=8)$ were numbered separately, and two random numbers were chosen from governmental,( Jiren and Jimma preparatory) schools and three from private (Eldana, Sos and Tesfa Tewahdo) secondary schools. In the second stage, students of selected governmental and private schools were stratified into grade levels from 9th to 12 th separately.

Finally, the sample size was allocated as per the number of students in each grade level using the proportional allocation method. After this, lists of students from 9th to 12th grade were obtained and the students were numbered consecutively in each class as per their roll numbers. This number was equally divided based on the number of sections in each class in a particular school.

One day before data collection selected adolescent students in the selected school were provided with information sheet about nature, purposes, benefits and adverse effects of the study and consent form paper to take to their parents at home who then signed consent forms if they 
allowed their child to participate in the study after they read information sheet. Students who are allowed to participate were given written informed ascent and signed for their participation.

\section{Independent variable/ Predictors}

Adverse childhood experiences Exposure to ACE was assessed using the ACE questionnaire (3), which addresses 10 item ACEs under three categories: 1. Abuse (emotional, physical and sexual), 2. Neglect (emotional and physical), and 3. Household dysfunction (parental separation/divorce, violence against mother, household substance abuse, household mental illness and incarceration of the house-hold member). The ACE questionnaire is a reliable and valid measure of childhood adversity that has been used extensively in large-scale ACE studies[30]. All questions about ACEs pertained to the respondents' first 18 years of life and were binary (yes vs. No). From these, variables were created to reflect any exposure to each ACE subtype (abuse, neglect, household dysfunction). We also calculated a total ACE score for each participant (+1 for each of the 10 types of ACE reported). Furthermore ACE score was treated as a categorical variable $(0,1-3$ or $>3)$ to capture any potential graded relationship with alcohol and chat use. This method of ACE score categorization has been used previously[12].

\section{Dependent variable /Outcomes}

The use of alcohol, tobacco and cigarette smoking was assessed by the following questions: "How many days did you smoke in the last 30 days?" and "How many days did you drink alcohol in the last 30 days?" If the adolescents had chew, smoked or had drunk alcohol at least once within the prior 30 days, they were considered as user of cigarette, tobacco or alcohol, respectively. These assessment was used by previous studies[31].

\section{Covariates}

In addition to socio-demographic variables each student also reported the Oslo 3-items social support scale was used to assess social support. A sum index was made by summarizing the raw scores, the sum ranging from 3 - 14[32].current depression symptoms were assessed by PHQ-9 a screening and diagnostic tool with cut point 5 or more symptoms out of 9 items[33]. The questionnaires were prepared in English then translated into Afaan Oromo and Amharic languages for better understanding. Ten second-year MSC psychiatry students were assigned to supervise and disseminate the questionnaires. Before the data collection, one-day training had been given for the supervisors. The supervisors for completeness and consistency of responses had checked the filled questionnaires daily. Before the actual data collection pre-test was made on $5 \%$ of the sample size in Agaro secondary school which is found in other zones near to the study site and necessary correction was taken after the pretest done on the questionnaires.

\section{Data processing and analysis}

Data were entered into Epi data 3.1 and exported to SPSS version 23.0. Chi-square tests were performed to examine any differences in ACE prevalence (cumulative and individual ACEs) by gender and by current substance use of alcohol and chat, and Logistic regression was used to compute adjusted odds ratios (ORs) and 95\% confidence intervals [34] to assess the association 
between ACEs and alcohol and chat use among adolescents. An analysis of variance was calculated using the ACE Score as a predictor, and alcohol and chat use as the dependent measure.

\section{RESULT}

\section{Description of socio-demographics}

Among the total number of 583 distributed questionnaires, 546 were filled completely and consistently making a response rate of $94.3 \%$. The remaining 37 questionnaires were not included in the study as a result of non-response rates collected from all the sampled schools. Of these, $442(81 \%)$ were studying in public schools and $104(19 \%)$ in private schools. Adolescent females outnumbered males. The majority of the participants were from the urban area. Their ages ranged from 14 to 19 years with a mean age of 16.83 years $(S D \pm 1.26)$. The majority of respondents 211(38.6\%) were under age 16, 153(28\%) were age 17 and 182(33.3) were above age 18. The majority (44\%) was Muslims and about (38.8\%) were Christians. Occupation of respondents' father was classified into four categories where 40 (7.3\%) were laborers, $219(40.1 \%)$ were merchants, 163 (29.9\%) were government employees, and $124(22.7 \%)$ private employees. Likewise, respondents' mother occupation was under the same category with an additional category namely being a housewife, which accounted for 135(24.7\%) mothers out of the total number of mothers. Five classifications were used to categorize respondents' father and mother educational levels. Accordingly, 150(27.5\%) of the response for fathers' educational level were under the certificate and above category while the number of mothers' educational level for the same category was 102 (18.7\%) (Table 1.)

\section{Description of Substance use among adolescents}

This study shows that significant number of students use chat chewing behavior $(26.6 \%)$ followed by alcohol use (20\%), and small number of student's use cigarette smoking and other illicit drug users (0.9\%) (ganja and Shisha) each(Table:1).

\section{Adverse Childhood Experience among adolescents}

Table: 2 shows among the 546 adolescents who participated in this study, 285(50.7\%) of the participants answered yes to at least one or more questions among the total 10 questions of ACEs. According to the three category of adverse childhood experience (ACE) from 332 female adolescents 60(18.1\%) had been abused either physically or sexually, 58(17.6\%) had been neglected and 100(30.4\%) had been household dysfunction. And also from 214 male adolescents $75(34.6 \%)$ had abused either physically or sexually, 43(19.6\%) had been neglected and $73(33.6 \%$ ) had been household dysfunction, (Table 2). 


\section{Description of Social support}

Measurement by the Oslo Three Items Social Support Scale revealed that 173(31.7\%) of respondents received poor social support, 211(38.6\%) of them had moderate social support while $162(29.7 \%)$ were have strong social support.

\section{Multivariate Relationships between ACEs and alcohol and chat consumption Risk Behaviors}

Regression models were estimated by entering the class variable and covariates simultaneously for each outcome variable, alcohol and chat use. Each ACEs class is compared to the reference group of Low ACEs (ACEs=0). Compared to ACEs=0, High/Multiple ACEs (ACEs=1-3 and above 3) was significantly associated with increased alcohol use behaviors (AOR=1.491(1.0723.078) and $(\mathrm{AOR}=3.171(1.330-7.560)$ respectively controlling other risk factors. similarly for chat use High/Multiple ACEs (ACEs=1-3 and above 3) was significantly associated with increased the chat use behaviors by 4.92 times $(\mathrm{AOR}=4.92,95 \% \mathrm{CI}=2.640-8.432)$ and 11.022 times $(\mathrm{AOR}=11.022,95 \% \mathrm{CI}=1.230-25.560)$ respectively.

In addition, we separately conducted a series of regression analyses to compare adolescents students in three types of ACEs (i.e., Abuse, Neglect and Household Dysfunction: (Table:5 )).

First, we found significant association with Neglect and Household Dysfunction which increases alcohol use by 1.137 times $(\mathrm{AOR}=1.137,95 \% \mathrm{CI}=1.094-3.175)$ and 3.028 times (AOR=3.028, $95 \% \mathrm{CI}=1.548-6.926)$ respectively. But in chat use behaviors we found significant association only with household dysfunction increasing it by 2.093times (AOR=2.093, 95\%CI=1.6294.897).

In examining the association between the ACEs and alcohol and chat use, male sex was a significant predictor. With being male increase alcohol and chat use by 2.603times ( $\mathrm{AOR}=2.603$, $95 \% \mathrm{CI}=1.657-4.089)$ and 1.312times $(\mathrm{AOR}=1.312,95 \% \mathrm{CI}=1.107-5.219)$ respectively.

In the model predicting alcohol use, father's educational status and current depression level were both significant predictors, that having grade 1-4 increases alcohol use by 7.247times ( $\mathrm{AOR}=7.247,95 \% \mathrm{CI}=1.604-32.691)$ as compared with illiterate. And current depression level increases alcohol use by 2.128times $(\mathrm{AOR}=2.128,95 \% \mathrm{CI}=1.055-8.291)$ as compared to nondepressed.

In a final model predicting chat use behaviors, residence was significant predictor, that coming from rural area of Jimma zone increases chat use by2.127times (AOR=2.127, 95\%CI=1.2137.361) as compared urban.

In a final model predicting alcohol and chat use behaviors, social support was significant predictor. Taking poor social support as reference, having moderate and strong social support decreases alcohol use by $35.1 \%(\mathrm{AOR}=0.649,95 \% \mathrm{CI}=0.364-0.956)$ and $78.1 \%(\mathrm{AOR}=0.219$, 
95\%CI=0.117-0.708) respectively And by $25.2 \%(\mathrm{AOR}=0.748,95 \% \mathrm{CI}=0.481-0.950)$ and $37.7 \% 9 \mathrm{AOR}=0.625,95 \% \mathrm{CI}=0.426-0.892)$ decreases chat use respectively.

\section{Discussion}

The findings of this study revealed that the commonly abused drugs among school going adolescents were alcohol 20\%, and chat 26.6\%. This finding alcohol use was slightly lower than study done in Northwest Ethiopia, Woreta town (59\%)but chat use was higher than these study, which was(13.8\%)[35].possible reason for the difference might be the culture difference of study population, that alcohol use was common in north part of Ethiopia(Woreta) and chat use was in eastern part(Jimma).prevalence of chat use was almost similar study done in eastern part of Ethiopia(Harare town) which was $24.2 \%$ (95\% CI 22.2\%-26.2\%)[36].

Most high school adolescents drink and chew because they want to experiment with alcohol, and chat, some drink and chew for the thrill of it, and others because it helps them relax. A new study finds that a fourth group of high school students share all those reasons for drinking, but they also drink and chew to get away from problems and to deal with anger or frustration issues and one these problem is ACEs[37, 38].

In the regression model alcohol use, the association between adverse childhood experiences and alcohol use behaviors were significant when other covariates were adjusted. Table 3 also shows that, Graded associations of cumulated adverse childhood experiences with the outcome variables were evident that the risk increases with ACEs count 13\%, 24.1\% and 37.7\% for ACEs=0, ACEs=1-3 and ACEs. $>3$ respectively . And also from the subtype we found significant association with Neglect and Household Dysfunction which increases alcohol use by 1.137 times $(\mathrm{AOR}=1.137,95 \% \mathrm{CI}=1.094-3.175)$ and 3.028 times $\quad(\mathrm{AOR}=3.028,95 \% \mathrm{CI}=1.548-6.926)$ respectively. This finding was similar with other studies done outside Ethiopia $[11,13,39,40]$.

These findings provide evidence that emotional, psychological and physical abuse, neglect and household dysfunction are stressful and traumatic factors in the childhood family environment are strongly associated with the initiation of alcohol use, particularly during early to midadolescence. ACEs influence the use of alcohol at an early age despite Ethiopian social and government efforts to delay its use through a variety of public health messages, and laws prohibiting alcohol use before age 21[29].

And also in the second regression model predicting chat use, we found similar finding as the first model that the association between adverse childhood experiences and chat use behaviors were significant when other covariates were adjusted. High/Multiple ACEs (ACEs=1-3 and above 3) increased chat use behaviors by 4.92 times $(\mathrm{AOR}=4.92,95 \% \mathrm{CI}=2.640-8.432)$ and 11.022 times ( $\mathrm{AOR}=11.022,95 \% \mathrm{CI}=1.230-25.560)$ respectively. And from subtype of ACEs household dysfunction increasing chat by 2.093times ( $\mathrm{AOR}=2.093,95 \% \mathrm{CI}=1.629-4.897)$. These finding was in line with other previous studies[41, 42]. 
In both regression models, our findings indicate that males have higher odds of drinking and chewing than females $(1.312 ; 95 \% \mathrm{CI}=(1.107-5.219)$ and $2.603 ; 95 \%=1.657-4.089)$ for alcohol and chat predicting models respectively. This is a common finding in alcohol and chat use studies where males use substances more and show higher tendency for dependence than females[43, 44].

And also social support was the only significant factor to decrease the risk of alcohol and chat use. Having moderate and strong social support compared with poor social support decreases alcohol use by $35.1 \%$ and $78.1 \%$ respectively. And by $23.2 \%$ and $37.5 \%$ decreases chat use when moderate and strong social support compared to poor social support respectively. This finding was in line with many other studies[45, 46]. Social supports as mediators were related to decreasing the effect of negative life events, specifically ACEs and deviant peer affiliations[47].

Furthermore in alcohol regression model, current level of depression and father educational level found to be significant. Having grade level (1-4) increases alcohol use by 7.247times $(\mathrm{AOR}=7.247,95 \% \mathrm{CI}=1.604-32.691)$ as compared with illiterate. In line with other study being educated decreases alcohol use that they will have good awareness than not educated[48, 49].

And current depression level increases alcohol use by 2.128times (AOR=2.128, 95\%CI=1.0558.291) as compared to non-depressed. As money other studies revealed depression can be risk and outcome of alcohol use[50].

Finally in the chat use regression model, residence found significant predictor of chat use. That coming from rural area of Jimma zone increases chat use by2.127times (AOR=2.127, 95\% CI=1.213-7.361) as compared urban. In line with study done in Ethiopia[50] access and tradition of rural area is more risk for chat use than the urban area. On the other hand studies done in Bahr Dar and Saudi Arabia[51, 52]. This may be due to study population difference; the more liberal life styles in urban areas compared to cultural conservatism in rural areas and may also be attributed to easiness of reporting chewing khat by urban adolescents than rural residents[52].

To our knowledge this is the one of the very few study to see association of ACEs and substance use (alcohol and chat) use among adolescent students. We used appropriate sampling procedure and analysis methods utilized were appropriate to the study and considered as the strength of the study. The study provides useful information that will inform policy makers to design a strategy to reduce the prevalence of ACEs and substance use (alcohol drinking and chat chewing) habit and its adverse social and health consequences.

This study was based on cross-sectional survey; causality cannot be inferred from our findings. Based on self-response for sensitive issues can invite social desirability bias and therefore underestimate utilization of alcohol drinking and chat chewing. The study was on school going adolescents; therefore impedes generalization to all adolescents in Ethiopia indicating a need for further study using a more representative sample of adolescents in the country 


\section{Conclusion and recommendation}

In conclusion, our findings indicate that there are high levels of alcohol drinking and chat chewing among the sampled school going adolescent students. ACEs are strongly associated with early initiation of alcohol and chat use among school going adolescents. Practical steps with families and guardians seek to reduce ACEs. Parents, as well as school and health authorities need to work more toward awareness creation about the hazards of alcohol drinking and chat chewing and the importance of social support to decrease the effects of past ACEs.

\section{Availability of data and materials}

The data used for this study can be made available with a reasonable request from the corresponding author.

\section{Abbreviations}

ACEs: Adverse childhood experiences

ACE-IQ : Adverse childhood experiences International Questionnaire

AOR: Adjusted odds ratio

AUD: Alcohol use disorder

AUDIT: Alcohol use disorder identification test

CI: Confidence interval

OR: Odds ratio

SUD: Substance use disorder

WHO: World Health Organization

\section{Declaration}

\section{Acknowledgements}

We would like to thank Jimma zone school administrators and students who gave us support and information. We also would like to thank MSC students of ICCMH for their genuine help, especially during the data collection and data entry of this study

\section{Funding}

This research was supported by Wollo University. The funder has no role in the interpretation of findings and decision for publication.

\section{Author information}

Affiliations 
Mekonnen Tsehay, Department of psychiatry, college of medicine and health science, Wollo University, Dessie, Ethiopia

Email: jimma1760@gmail.com

Mogese Necho, department of psychiatry, college of medicine and health science, Wollo University, Dessie, Ethiopia

Email: nechomoges2014@gmail.com

Asmare Belete. Department of psychiatry, college of medicine and health science, Wollo University, Dessie, Ethiopia

Email: yasmarebel@yahoo.com

\section{Contributions}

MT conceived and designed the study. MT, MN and $\mathrm{AB}$ analyzed the data and drafted the manuscript. All the authors read the manuscript several times and have given their final approval for publication.

\section{Corresponding author}

Correspondence to Mekonnen Tsehay, Department of psychiatry, college of medicine and health science, Wollo University, Dessie, Ethiopia. Email: jimma 1760@ gmail.com , PO.BOX: 1145

\section{Ethics declarations}

Ethics approval and consent to participate

We obtained ethical approval from the Ethical Review Committee of the College of Health Sciences, Jimma University. We obtained further permission to conduct the research from the respective Jimma zone educational office. Written informed consent and ascent was obtained from each participant. We kept privacy and confidentiality by replacing all names with codes and by keeping all the data we collected in a locked bag.

\section{Consent for publication}

Not applicable.

\section{Competing interests}

The authors declare that they have no conflict of interest. 


\section{REFERENCE}

1. Denton DA. National Health and Medical Research Council (NHMRC) 2009. 2009.

2. Moore E, Coffey C, Carlin JB, Alati R, Patton GC. Assessing alcohol guidelines in teenagers: results from a 10-year prospective study. Australian and New Zealand journal of public health. 2009;33(2):154-9.

3. Degenhardt L, Stockings E, Patton G, Hall WD, Lynskey M. The increasing global health priority of substance use in young people. The Lancet Psychiatry. 2016;3(3):251-64.

4. Simons-Morton B, Haynie D, Liu D, Chaurasia A, Li K, Hingson R. The effect of residence, school status, work status, and social influence on the prevalence of alcohol use among emerging adults. Journal of studies on alcohol and drugs. 2016;77(1):121-32.

5. Kuntsche E, Rossow I, Simons-Morton B, Bogt TT, Kokkevi A, Godeau E. Not Early Drinking but Early Drunkenness Is a Risk Factor for Problem Behaviors Among Adolescents from $38 \mathrm{E}$ uropean and $\mathrm{N}$ orth A merican Countries. Alcoholism: Clinical and experimental research. 2013;37(2):308-14.

6. Degenhardt L, Glantz M, Evans-Lacko S, Sadikova E, Sampson N, Thornicroft G, et al. Estimating treatment coverage for people with substance use disorders: an analysis of data from the World Mental Health Surveys. World Psychiatry. 2017;16(3):299-307.

7. Organization WH. Global status report on alcohol and health 2018: World Health Organization; 2019. 
8. Monti PM, Miranda Jr R, Nixon K, Sher KJ, Swartzwelder HS, Tapert SF, et al. Adolescence: booze, brains, and behavior. Alcoholism: Clinical and Experimental Research. 2005;29(2):207-20.

9. Witt ED. Research on alcohol and adolescent brain development: opportunities and future directions. Alcohol. 2010;44(1):119-24.

10. Olawole-Isaac A, Ogundipe O, Amoo EO, Adeloye D. Substance use among adolescents in sub-Saharan Africa: A systematic review and meta-analysis. South African Journal of Child Health. 2018;12(SPE):s79-s84.

11. Duke NN, Pettingell SL, McMorris BJ, Borowsky IW. Adolescent violence perpetration: associations with multiple types of adverse childhood experiences. Pediatrics. 2010;125(4):e778e86.

12. Anda RF, Croft JB, Felitti VJ, Nordenberg D, Giles WH, Williamson DF, et al. Adverse childhood experiences and smoking during adolescence and adulthood. JAMA. 1999;282(17):1652-8.

13. Dube SR, Miller JW, Brown DW, Giles WH, Felitti VJ, Dong M, et al. Adverse childhood experiences and the association with ever using alcohol and initiating alcohol use during adolescence. Journal of Adolescent Health. 2006;38(4):444. e1-. e10.

14. Hughes K, Bellis MA, Hardcastle KA, Sethi D, Butchart A, Mikton C, et al. The effect of multiple adverse childhood experiences on health: a systematic review and meta-analysis. The Lancet Public Health. 2017;2(8):e356-e66.

15. Pereda N, Guilera G, Forns M, Gómez-Benito J. The prevalence of child sexual abuse in community and student samples: A meta-analysis. Clinical psychology review. 2009;29(4):32838.

16. Stoltenborgh M, Bakermans-Kranenburg MJ, Alink LR, Van Ijzendoorn MH. The universality of childhood emotional abuse: a meta-analysis of worldwide prevalence. Journal of Aggression, Maltreatment \& Trauma. 2012;21(8):870-90.

17. Jansen HA, Watts C, Ellsberg M, Heise L, Garcia-Moreno C. Interviewer training in the WHO multi-country study on women's health and domestic violence. Violence against women. 2004;10(7):831-49.

18. Bartley M. Life gets under your skin: UCL, Research Departement of Epidemiology and Public Health; 2012.

19. Anda R. The health and social impact of growing up with adverse childhood experiences: The human and economic costs of the status quo. Centers for Disease Control and Prevention. 2007.

20. Campbell JA, Walker RJ, Egede LE. Associations between adverse childhood experiences, high-risk behaviors, and morbidity in adulthood. American journal of preventive medicine. 2016;50(3):344-52.

21. Hillis SD, Anda RF, Felitti VJ, Marchbanks PA. Adverse childhood experiences and sexual risk behaviors in women: a retrospective cohort study. Family planning perspectives. 2001:206-11.

22. Bellis MA, Lowey H, Leckenby N, Hughes K, Harrison D. Adverse childhood experiences: retrospective study to determine their impact on adult health behaviours and health outcomes in a UK population. Journal of public health. 2014;36(1):81-91.

23. Forster M, Grigsby TJ, Rogers CJ, Benjamin SM. The relationship between family-based adverse childhood experiences and substance use behaviors among a diverse sample of college students. Addictive behaviors. 2018;76:298-304. 
24. Allem J-P, Soto DW, Baezconde-Garbanati L, Unger JB. Adverse childhood experiences and substance use among Hispanic emerging adults in Southern California. Addictive behaviors. 2015;50:199-204.

25. Lee K, Pang YC, Lee JAL, Melby JN. A study of adverse childhood experiences, coping strategies, work stress, and self-care in the child welfare profession. Human Service Organizations: Management, Leadership \& Governance. 2017;41(4):389-402.

26. Richard A, Rohrmann S, Vandeleur CL, Schmid M, Barth J, Eichholzer M. Loneliness is adversely associated with physical and mental health and lifestyle factors: Results from a Swiss national survey. PLoS One. 2017;12(7).

27. Armstrong MI, Birnie-Lefcovitch S, Ungar MT. Pathways between social support, family well being, quality of parenting, and child resilience: What we know. Journal of child and family studies. 2005;14(2):269-81.

28. Blum RW, Li M, Naranjo-Rivera G. Measuring adverse child experiences among young adolescents globally: Relationships with depressive symptoms and violence perpetration. Journal of Adolescent Health. 2019;65(1):86-93.

29. Reda AA, Moges A, Wondmagegn BY, Biadgilign S. Alcohol drinking patterns among high school students in Ethiopia: a cross-sectional study. BMC public health. 2012;12(1):213.

30. Ho GW, Chan AC, Chien W-T, Bressington DT, Karatzias T. Examining patterns of adversity in Chinese young adults using the Adverse Childhood Experiences-International Questionnaire (ACE-IQ). Child abuse \& neglect. 2019;88:179-88.

31. Gonçalves H, Soares ALG, Santos APGd, Ribeiro CG, Bierhals IO, Vieira LS, et al. Adverse childhood experiences and consumption of alcohol, tobacco and illicit drugs among adolescents of a Brazilian birth cohort. Cadernos de saude publica. 2016;32:e00085815.

32. Kocalevent R-D, Berg L, Beutel ME, Hinz A, Zenger M, Härter M, et al. Social support in the general population: standardization of the Oslo social support scale (OSSS-3). BMC psychology. 2018;6(1):31.

33. Gelaye B, Williams MA, Lemma S, Deyessa N, Bahretibeb Y, Shibre T, et al. Validity of the patient health questionnaire-9 for depression screening and diagnosis in East Africa. Psychiatry research. 2013;210(2):653-61.

34. Rosenberg KP, Bleiberg KL, Koscis J, Gross C. A survey of sexual side effects among severely mentally ill patients taking psychotropic medications: impact on compliance. Journal of Sex \&Marital Therapy. 2003;29(4):289-96.

35. Birhanu AM, Bisetegn TA, Woldeyohannes SM. High prevalence of substance use and associated factors among high school adolescents in Woreta Town, Northwest Ethiopia: multidomain factor analysis. BMC public health. 2014;14(1):1186.

36. Reda AA, Moges A, Biadgilign S, Wondmagegn BY. Prevalence and determinants of khat (Catha edulis) chewing among high school students in eastern Ethiopia: a cross-sectional study. PLoS One. 2012;7(3).

37. Copeland LA, Shope JT, Waller PF. Factors in adolescent drinking/driving: binge drinking, cigarette smoking, and gender. Journal of School Health. 1996;66(7):254-60.

38. Ellickson PL, McGUIGAN KA, Adams V, Bell RM, Hays RD. Teenagers and alcohol misuse in the United States: by any definition, it's a big problem. Addiction. 1996;91(10):1489503.

39. Kim YH. Associations of adverse childhood experiences with depression and alcohol abuse among Korean college students. Child abuse \& neglect. 2017;67:338-48. 
40. Dube SR, Anda RF, Felitti VJ, Edwards VJ, Croft JB. Adverse childhood experiences and personal alcohol abuse as an adult. Addictive behaviors. 2002;27(5):713-25.

41. Kiburi SK, Molebatsi K, Obondo A, Kuria MW. Adverse childhood experiences among patients with substance use disorders at a referral psychiatric hospital in Kenya. BMC psychiatry. 2018;18(1):197.

42. Belew M, Kebede D, Kassaye M, Enquoselassie F. The magnitude of khat use and its association with health, nutrition and socio-economic status. Ethiopian Medical Journal. 2000;38(1):11-26.

43. Odenwald M, Neuner F, Schauer M, Elbert T, Catani C, Lingenfelder B, et al. Khat use as risk factor for psychotic disorders: a cross-sectional and case-control study in Somalia. BMC medicine. 2005;3(1):5.

44. Aldridge RW, Story A, Hwang SW, Nordentoft M, Luchenski SA, Hartwell G, et al. Morbidity and mortality in homeless individuals, prisoners, sex workers, and individuals with substance use disorders in high-income countries: a systematic review and meta-analysis. The Lancet. 2018;391(10117):241-50.

45. Wills TA, Vaughan R. Social support and substance use in early adolescence. Journal of behavioral medicine. 1989;12(4):321-39.

46. Wills TA, Cleary SD. How are social support effects mediated? A test with parental support and adolescent substance use. Journal of personality and social psychology. 1996;71(5):937.

47. Piko B. Perceived social support from parents and peers: Which is the stronger predictor of adolescent substance use? Substance use \& misuse. 2000;35(4):617-30.

48. Chaffin M, Silovsky JF, Funderburk B, Valle LA, Brestan EV, Balachova T, et al. Parent-child interaction therapy with physically abusive parents: efficacy for reducing future abuse reports. Journal of consulting and clinical psychology. 2004;72(3):500.

49. Reyno SM, McGrath PJ. Predictors of parent training efficacy for child externalizing behavior problems-a meta-analytic review. Journal of child psychology and psychiatry. 2006;47(1):99-111.

50. Conner KR, Pinquart M, Gamble SA. Meta-analysis of depression and substance use among individuals with alcohol use disorders. Journal of substance abuse treatment. 2009;37(2):127-37.

51. Mulugeta Y. Khat chewing and its associated factor among college students in Bahir Dar Town, Ethiopia. Science Journal of Public Health. 2013;1(5):209-14.

52. Ageely HM. Prevalence of Khat chewing in college and secondary (high) school students of Jazan region, Saudi Arabia. Harm Reduction Journal. 2009;6(1):11.

List of table

Table 1: Socio demographic profile of adolescents who participated in the study ..... Error! Bookmark not defined.

Table 2: cross tabulation of ACEs distribution by sex of respondents. Error! Bookmark not defined. Table 3: Effect of ACEs count on alcohol use symptoms with cross tabulation Error! Bookmark not defined. 
Table 4: Binary and multivariate logistic regression examining the association between predictors and chat use, April 2020 Error! Bookmark not defined. Table 5: Binary multivariate logistic regression examining the association between predictors and alcohol use, April 2020. Error! Bookmark not defined. 